\title{
Characteristics of the motor responsible for the gliding of native
}

\section{microtubules from squid axoplasm}

\author{
DIETER G. WEISS ${ }^{1,2,3}$, DIETER SEITZ-TUTTER ${ }^{1,2,3}$ and GEORGE M. LANGFORD ${ }^{2,3,4}$ \\ ${ }^{1}$ Institut für Zoologie, Technische Universität München, D-8046 Garching, Germany \\ ${ }^{2}$ Marine Biological Laboratory, Woods Hole, MA 02543, USA \\ ${ }^{3}$ Stazione Zoologica Antonio Dohrn, Villa Comunale, Napoli, Italy \\ ${ }^{4}$ Department of Physiology, School of Medicine, University of North Carolina, Chapel Hill, NC 27514, USA
}

\begin{abstract}
Summary
Nucleotide-dependent movement of native microtubules (nMTs) in squid axoplasm has biochemical and biophysical characteristics of kinesin-driven motility. However, the high vanadate and $N$-ethylmaleimide sensitivity and the velocity demonstrate that the properties of the native motile system differ considerably from those of purified kinesin preparations.
\end{abstract}

Key words: native microtubules, squid axoplasm, gliding assay, kinesin, dynein.

\section{Introduction}

The ATP-dependent gliding movement of microtubules over a glass surface was first observed with native microtubules (nMTs) dissociated from extruded squid axoplasm (Allen and Weiss, 1985; Allen et al. 1985). Subsequently, gliding motility of MTs was used extensively as an assay for the characterization of putative organelle translocators, i.e. kinesin and cytoplasmic dynein (e.g. Vale et al. 1985a,c; Paschal et $a l$. 1987). In studies using purified motor proteins, similar patterns of movement of in vitro reconstituted MTs have been reported, but considerable biochemical and pharmacological differences can be demonstrated to occur in these two model systems. It is the aim of this report to summarize our studies on the motile behaviour of nMTs and to correlate some of the pharmacological properties of $n M T$ gliding with those of organelle transport along the MTs and those' purified motors. Such studies aim at determining which force-generating enzymes are involved in nMT gliding.

\section{Results and discussion}

After the axoplasm is extruded from the axolemma and the cell layer attached to it, numerous nMTs begin to dissociate from the remaining bulk axoplasm. The techniques used to obtain nMTs and to observe their motile behaviour reproducibly have been published in detail (Allen et al. 1985; Weiss et al. 1989; Weiss et al. 1990).

Many of these nMTs or nMT fragments, which range in length from 1 to $30 \mu \mathrm{m}$ (mean \pm s.D.: $12.5 \pm 6.1 \mu \mathrm{m}$ ), attach to the glass surface and glide along it. Short nMTs $(<10 \mu \mathrm{m})$ are usually straight and glide along a linear Journal of Cell Science, Supplement 14, 157-161 (1991)

Printed in Great Britain (C) The Company of Biologists Limited 1991 path, while longer nMTs tend to bow. In the latter case, it is frequently observed that the front and rear ends do not follow the same path but show slight lateral deviations.

When the forward progress of the nMT's frontal end is fully blocked, by immobilization of a portion of the MT, a rhythmic bending of the MT tail end commences, a process we called 'fishtailing' (Fig. 1) (Allen et al. 1985; Vale et al. 1985b; Pryer et al. 1986). This planar movement resembles the propagation of bending waves on flagella and can be simulated mathematically in a similar way (Brokaw, 1986).

If the microtubule is fixed only at a single attachment point near the frontal end, the force pushing from the rear end will produce a planar spiraling motion (Fig. 2). We have shown that all three patterns of motility (gliding, fishtailing and spiraling) are produced by the same mechanism, since their speeds and frequencies either correlate closely or are the same in a given preparation (Fig. 3; Seitz-Tutter, 1990; D. Seitz-Tutter, D. G. Weiss and G. M. Langford, unpublished observations).

Large organelles that are transported retrogradely in the intact axon, move on gliding nMTs toward their frontal ends, while the small vesicles move in the opposite direction (Allen et al. 1985). In addition, in nMTs showing dynamic instability, only the rear end exhibits detectable length changes (Seitz-Tutter et al. 1988; Weiss et al. 1988). On the basis of these findings we concluded that the frontal end of a gliding nMT corresponds to its slow growing or minus end. The same conclusion was obtained from their studies by Vale et al. (1985b).

Quantitative computer-based motion analysis of the gliding behaviour of nMTs (Seitz-Tutter, 1990; D. SeitzTutter, D. G. Weiss and G. M. Langford, unpublished observations) revealed that only displacement in the main direction of movement (along the MT's longitudinal axis) contributes to the MT's net translocation: the component perpendicular to it is negligible. The movement of nMTs is smooth and continuous; Fast Fourier Transform analysis did not yield evidence for oscillatory or otherwise periodic components of the motion. The gliding velocity of nMTs, which was shown to be independent of their length, is in the range of $0.25-0.70 \mu \mathrm{m} \mathrm{s}^{-1}$; the velocity histogram shows a Gaussian and unimodal distribution (mean \pm s.D.: $0.45 \pm 0.12 \mu \mathrm{m} \mathrm{s}^{-1}$ ). We were able to establish a statistically significant linear correlation between the velocity of endogenous organelles transported to the plus end of a gliding nMT (anterogradely) and the gliding velocity of the corresponding MT $(r=0.89)$. For retrogradely trans- 
ported organelles, however, such a correlation was absent (Seitz-Tutter, 1990; D. Seitz-Tutter, D. G. Weiss and G. M. Langford, unpublished observations).

The pharmacological properties of the gliding behaviour of nMTs (Seitz-Tutter, 1990; D. Seitz-Tutter, D. G. Weiss and G. M. Langford, unpublished observations) show the following similarities with MT-motility in reconstituted systems containing purified kinesin (Cohn et al. 1987; Scholey et al. 1985; Vale et al. 1985a,c): gliding of nMTs is inhibited by apyrase $\left(0.5 \mathrm{mg} \mathrm{ml}^{-1}\right)$, AMP-PNP and PPP

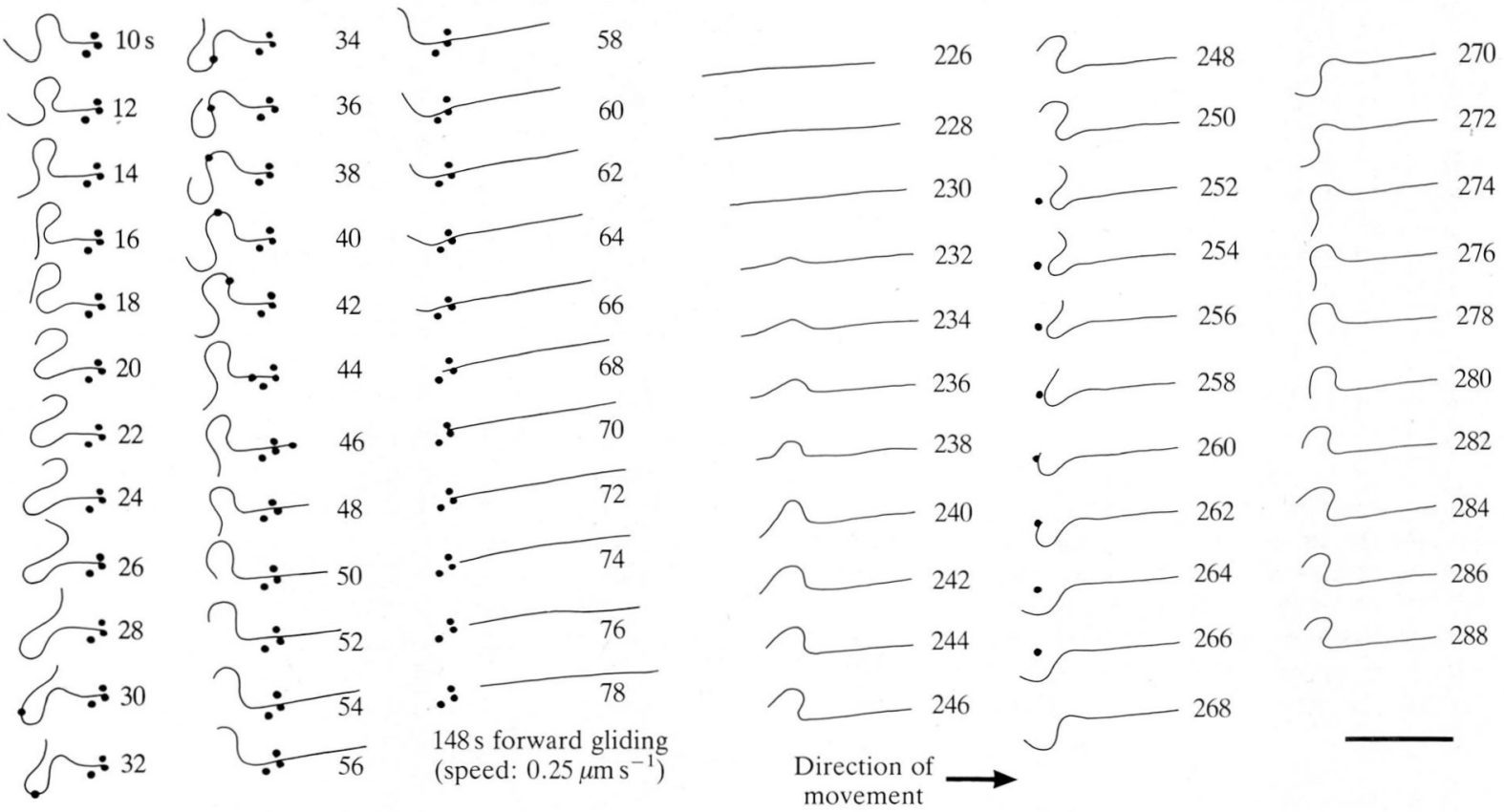

Fig. 1. Gliding and fishtailing behaviour of nMT. For the first $34 \mathrm{~s}$ the straight gliding movement of this nMT is hindered by an invisible obstacle. The force-generating enzymes cause the fishtailing motion until the MT becomes free from the obstacle (44 s). The MT straightens and glides between second 78 and 226 toward the right (speed $0.25 \mu \mathrm{m} \mathrm{s}^{-1}$ ) until the frontal end becomes again attached by an obstacle or a 'sticky' site on the surface and the tail end displays the fishtailing behaviour again. The particle which is transported along the microtubule between time points 30 and 44 does not interfere with the fishtailing motion, but probably causes the microtubule to resume gliding by releasing the front end from its attachment. The numbers indicate the elapsed time in seconds. Bar $5 \mu \mathrm{m}$.<smiles>O=C1CCCCC1</smiles>

0:03<smiles></smiles>

0:09-0:16

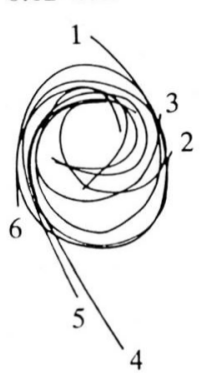

0:04<smiles></smiles>

0:05;

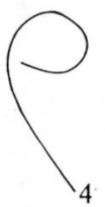

0:08

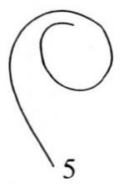

0:09

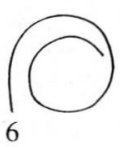

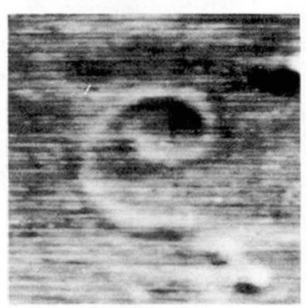

0:24-0:32

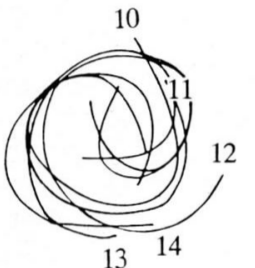

$0: 32-0: 45$

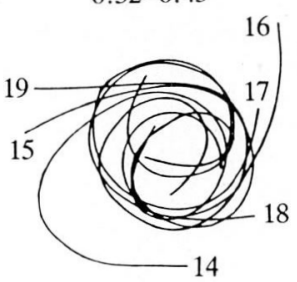

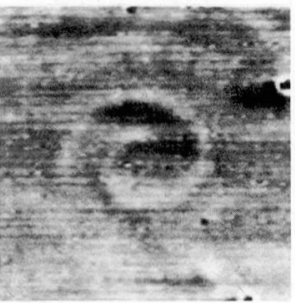

Fig. 2. Spiraling motion of nMT. A sequence of shapes assumed by a microtubule which is bent into a planar spiral and rotates. The length of this microtubule is $11 \mu \mathrm{m}$. The consecutive shapes are drawn at $1 \mathrm{~s}$ intervals and were superimposed in the lower panels to show that the microtubule is not moving on a defined track on the glass surface (the small numbers indicate the rear, pushing end). Instead, it appears as if the microtubule can move equally well over the entire area. The observation time in the top row is $8 \mathrm{~s}$ corresponding to about $3 / 4$ of a full turn (time is given in min:s). Two representative videomicrographs of this MT are included. 


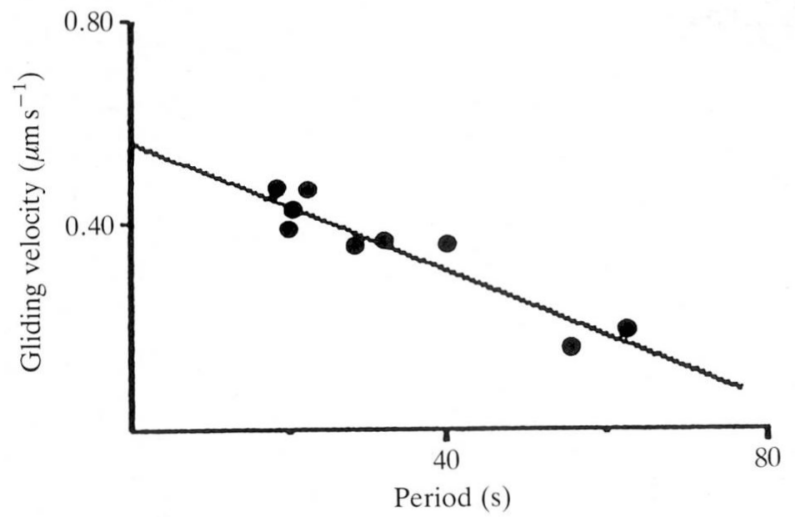

Fig. 3. Correlation between beating frequency and gliding velocity. The period of the fishtailing motion is independent of the length of the motile part of the MT but highly correlated with the mean gliding velocities of other MTs in the same preparations ( $r=0.94, P=0.01$, Pearson-Bravais test). This result supports the notion that gliding, fishtailing, and rotating movements are all driven by the same mechanism. The point of intersection of the regression line with the ordinate $\left(0.58 \mu \mathrm{m} \mathrm{s}^{-1}\right)$ matches the maximum gliding velocity of MTs observed in the extruded axoplasm preparations (mean: $0.45 \pm 0.04 \mu \mathrm{m} \mathrm{s}^{-1}$, max.: $0.65 \mu \mathrm{m} \mathrm{s}^{-1}$ ). when in excess of ATP. But, in contrast to the movement of reassembled MTs induced by purified kinesin, the gliding of nMTs was partially inhibited by $1 \mathrm{~mm}$ $N$-ethylmaleimide (NEM) and completely blocked by $2 \mathrm{~mm}$ (Table 1). Furthermore, the straight gliding of nMTs was significantly inhibited by relatively low concentrations of vanadate $(20 \mu \mathrm{M})$ that specifically inhibit dynein-like, but not kinesin-like, ATPases. Gliding was blocked completely in the presence of $50 \mu \mathrm{M}$ vanadate.

These findings are in contrast to reports on kinesininduced gliding in in vitro reconstituted systems, where inhibition occurs usually only at $50-400 \mu \mathrm{M}$ vanadate (for references see Table 1). In the presence of $50 \mu \mathrm{M}$ vanadate, retrograde organelle transport along nMTs was already completely blocked, whereas anterograde organelle transport continued almost undisturbed for about $30 \mathrm{~min}$ before a decline in velocity was detected. After replacement of the vanadate-containing buffer by vanadate-free medium only, anterograde organelle transport was reactivated. Gliding of nMTs is therefore sensitive to even lower concentrations of vanadate than retrograde transport (Table 1).

The sensitivity to various inhibitors of the ATPase activity and of the microtubule translocation is well known, for both purified kinesin and cytoplasmic dynein. In such preparations kinesin was found to be less sensitive than dynein for NEM inhibition (2-5 mM versus

Table 1. Properties of motors and MT motility in vivo and in vitro

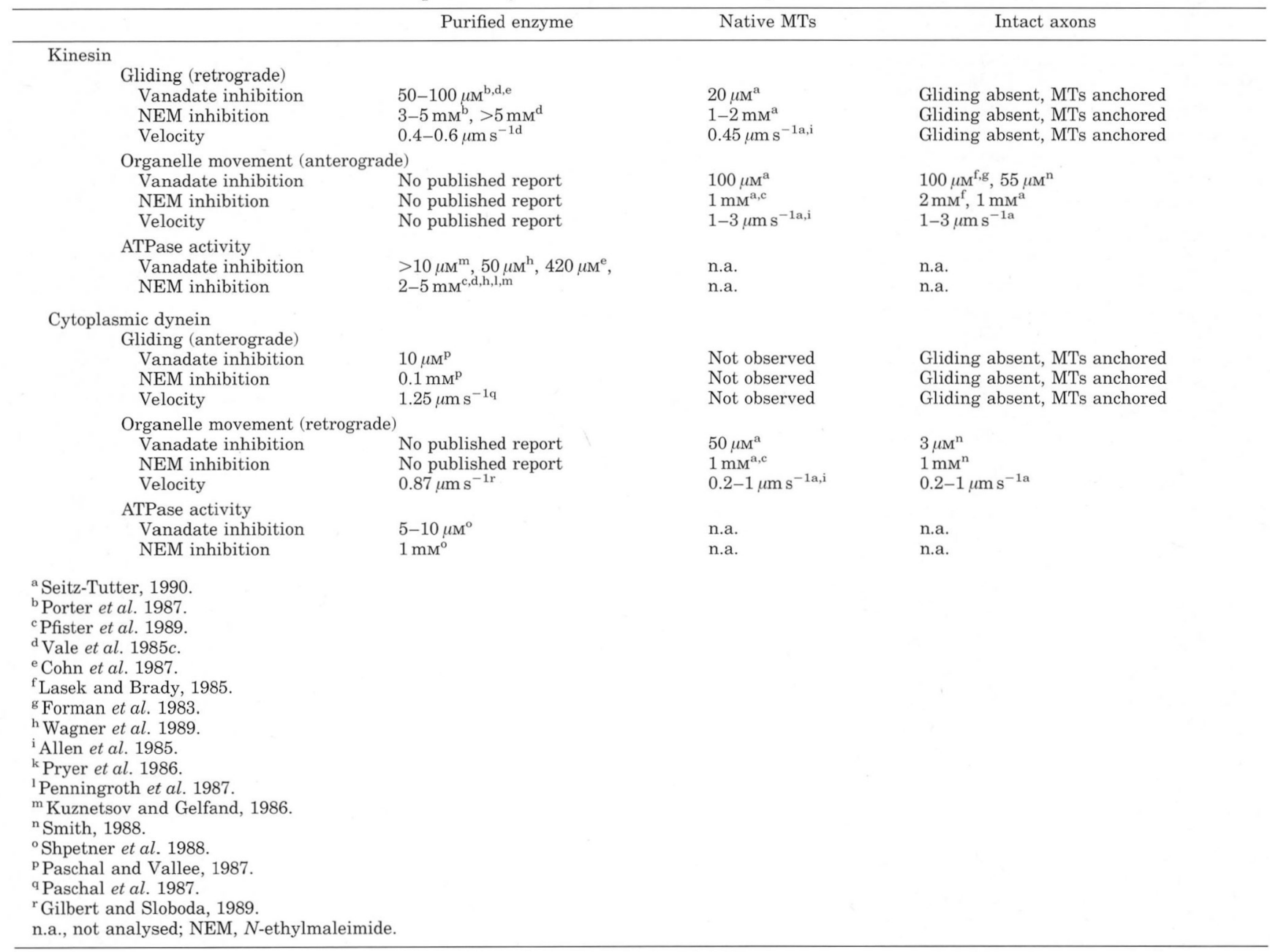


0.1-1 mM, respectively) and for vanadate inhibition (20-400 $\mu \mathrm{M}$ versus 5-10 $\mu \mathrm{M}$, respectively) (Table 1 ). In intact axons the same difference was observed for the effect of vanadate on organelle transport in the two directions (retrograde transport is inhibited by $3 \mu \mathrm{M}$ vanadate, anterograde transport by $50-100 \mu \mathrm{M}$ ), but it was found that NEM inhibited both directions similarly (1-2 mM) (Table 1). This situation is very similar to our findings on the pharmacology of organelle transport along nMTs (Table 1). The major surprise is, however, the finding that gliding of nMTs is even more sensitive than retrograde organelle movement.

Taken together, gliding of nMTs has mainly kinesin-like properties: (1) the direction is the same as that of the motor for anterograde vesicle transport, (2) the velocity of anterograde transport and $\mathrm{nMT}$ gliding correlate closely (although the absolute values are different) (Allen $e t$ al. 1985). Not fully compatible with the hypothesis that kinesin is the motor for nMT motility and yet to be resolved are the following findings: (1) the velocity of purified kinesin is much slower than anterograde vesicle transport and nMT gliding, (2) the sensitivity to NEM and vanadate of nMT gliding is higher than that of purified kinesin and resembles to some extent that of purified dynein or retrograde organelle transport, (3) Leopold et al. (1990) reported that the nucleotide specificity of anterograde organelle motility in the native system differs considerably from the properties of purified kinesin.

However, the majority of the findings support the notion that the motility of nMTs is driven by kinesin, the motor of anterograde transport. Although retrograde motors were certainly present, since we observed minus end-directed organelle transport in all our preparations, there was no indication of plus end-directed MT gliding caused by cytoplasmic dynein. A similar situation is known from in vitro experiments in which both motors were simultaneously present (Lye et al. 1989).

The discrepancies in the pharmacological properties of nMT gliding and anterograde organelle transport may indicate different states of the motor. In the case of gliding the kinesin molecules are attached to the glass surface perhaps in an unspecific way and may even be partially denatured. In the case of vesicle transport the conformational state of kinesin may be well defined and determined by its interaction with specific receptors or perhaps with dynein residing nearby at the organelle membrane (compare Brady et al. 1990; Sheetz et al. this volume).

The study of nMTs can therefore be used to bridge the gap between the relatively different results obtained with intact axons and with purified motors. Moreover, it appears that knowledge of the detailed properties of nMT motility is necessary, in addition to the study of purified motors, in order to understand fully the molecular mechanism of MT-dependent organelle transport.

The support from NSF Grant BNS-9004526, NATO Research Grant CRG(30)0874/86 (GML), and DFG grant We 790/12 (DGW) is gratefully acknowledged. We thank W. Maile for his help in providing Figs 1 and 2.

\section{References}

Allen, R. D. And Weiss, D. G. (1985). An experimental analysis of the mechanisms of fast axonal transport in the squid giant axon. In Cell Motility: Mechanism and Regulation (ed. H. Ishikawa, S. Hatano and H. Sato), vol. 10, pp. 327-333. Yamada Conference, Sept. 1984, Tokyo: University of Tokyo Press.
Allen, R. D., Weiss, D. G., Hayden, J. H., Brown, D. T., Fujiwake, H. AND SIMPSON, M. (1985). Gliding movement of and bidirectional organelle transport along single native microtubules from squid axoplasm: evidence for an active role of microtubules in cytoplasmic transport. J. Cell Biol. 100, 1736-1752.

Brady, S. T., Pfister, K. K. and Bloom, G. S. (1990). A monoclonal antibody against kinesin inhibits both anterograde and retrograde fast axonal transport in squid axoplasm. Proc. natn. Acad. Sci. U.S.A. 87, 1061-1065.

BroKaw, C. J. (1986). Computer simulation of bend propagation by axoplasmic microtubules. Cell Motil. Cytoskel. 6, 347-353.

Cohn, S. A., Ingold, A. L. AND Scholey, J. M. (1987). Correlation between the ATPase and microtubule translocating activities of sea urchin kinesin. Nature 328, 1160-1163.

Forman, D. S., Brown, K. J. and Livengood, D. R. (1983). Fast axonal transport in permeabilized lobster giant axon is inhibited by vanadate. $J$. Neurosci. 3, 1279-1288.

Gilbert, S. P. AND Sloboda, R. D. (1989). A squid dynein isoform promotes axoplasmic vesicle translocation. J. Cell Biol. 109 2379-2394.

Kuznetsov, S. A. AND Gelfand, V. I. (1986). Bovine brain kinesin is a microtubule-activated ATPase. Proc. natn. Acad. Sci. U.S.A. 83, 8530-8534.

LASEK, R. J. AND BRADY, S. T. (1985). Attachment of transported vesicles to microtubules in axoplasm is facilitated by AMP-PNP. Nature $\mathbf{3 1 6}$, $645-647$.

Leopold, P. L., Snyder, R., Bloom, G. S. and Brady, S. T. (1990). Nucleotide specificity for the bidirectional transport of membranebounded organelles in isolated axoplasm. Cell Motil. Cytoskel. 15, 210-219.

Lye, R. J., Pfarr, C. M. and Porter, M. E. (1989). Cytoplasmic dynein and microtubule translocators. In Cell Movement, Volume 2: Kinesin Dynein, and Microtubule Dynamics (ed. F. Warner and R. McIntosh), pp. 141-201. A. R. Liss, New York

Paschal, B. M., ShPetiner, H. S. ANd Vallee, R. B. (1987). MAP 1C is a microtubule-activated ATPase which translocates microtubules in vitro and has dynein-like properties. J. Cell Biol. 105, 1273-1283.

PAschal, B. M. AND VALLeE, R. B. (1987). Retrograde transport by the microtubule-associated protein MAP 1C. Nature 330, 181-183.

Penningroth, S. M., Rose, P. M. and Peterson, D. D. (1987). Evidence that the $116 \mathrm{kDa}$ component of kinesin binds and hydrolyzes ATP. FEBS Lett. 222, 204-210.

Pfister, K. K., Wagner, M. C., Bloom, G. S. and Brady, S. T. (1989). Modification of the microtubule-binding and ATPase activities of kinesin by $N$-Ethylmaleimide (NEM) suggests a role for sulfhydryls in fast axonal transport. Biochemistry 28, 9006-9012.

Porter, M. E., Scholey, J. M., Stemple, D. L., Vigers, G. P. A., Vale, R. D., SheEtz, M. P. AND McIntosh, J. R. (1987). Characterization of the microtubule movement produced by sea urchin egg kinesin. J. biol. Chem. 262, 2794-2802.

Pryer, N. K., Wadsworth, P. and Salmon, E. D. (1986). Polarized microtubule gliding and particle saltation produced by soluble factors from sea urchin eggs and embryos. Cell Motil. Cytoskel. 6, 537-548.

Scholey, J. M., Porter, M. E., Grissom, P. M. AND McIntosh, J. R. (1985). Identification of kinesin in sea urchin eggs and evidence for its localization in the mitotic spindle. Nature 318, 483-486.

Seitz-Tutrer, D. (1990). Organellentransport und Eigenbewegung der Mikrotubuli im Axoplasma des Tintenfisch-Riesenaxons. Ph. D. Thesis, University of Munich.

Seitz-Tutter, D., Langford, G. M. and Weiss, D. G. (1988). Dynamic instability of native microtubules from squid axon is rare and independent of gliding and vesicle transport. Expl Cell Res. 178, 504-511.

Shpetner, H. S., Paschal, B. M. and Vallee, R. B. (1988). Characterization of the microtubule-activated ATPase of brain cytoplasmic dynein (MAP1C). J. Cell Biol. 107, 1001-1009.

SMITH, R. S. (1988). Studies on the mechanism of the reversal of rapid organelle transport in myelinated axons of Xenopus laevis. Cell Motil. Cytoskel. 10, 296-308.

VAle, R. D., ReEse, T. S. AND Sheetz, M. P. (1985c). Identification of a novel, force-generating protein, kinesin, involved in microtubule-based motility. Cell 42, 39-50.

Vale, R. D., Schnapp, B. J., Mitchison, T., Steder, E., Reese, T. S. AND SheEtz, M. P. (1985b). Different axoplasmic proteins generate movement in opposite directions along microtubules in vitro. Cell $\mathbf{4 3}$ $623-632$

Vale, R. D., Schnapp, B. J., Reese, T. S. and Sheetz, M. P. (1985a). Organelle, bead and microtubule translocations promoted by soluble factors from the squid giant axon. Cell 40, 559-569.

Wagner, M. C., Pfister, K. K., Bloom, G. S. and Brady, S. T. (1989). Copurification of kinesin polypeptides with microtubule-stimulated 
$\mathrm{Mg}$-ATPase activity and kinetic analysis of enzymatic properties. Cell Motil. Cytoskel. 12, 195-215.

Weiss, D. G., Langford, G. M., Seitz-Tutter, D. and Keller, F. (1988). Dynamic instability and motile events of native microtubules from squid axoplasm. Cell Motil. Cytoskel. 10, 285-296.

Weiss, D. G., MaILE, W. AND Wick, R. A. (1989). Video microscopy. In
Light Microscopy in Biology. A Practical Approach (ed. A. J. Lacey), pp. 221-278. IRL Press, Oxford.

Weiss, D. G., Meyer, M. A. And Langford, G. M. (1990). Studying axoplasmic transport using video microscopy and the squid giant axon. In Squid as Experimental Animals (ed. D. L. Gilbert, W. J. Adelman and J. Arnold), pp. 303-321. Plenum Press, New York. 
